\title{
Plasmids pIP419 and pIP421 from Bacteroides: 5-nitroimidazole resistance genes and their upstream insertion sequence elements
}

\author{
Stephanie Trinh, Abdellatif Haggoud, $\uparrow$ Gilles Reysset and Madeleine \\ Sebald
}

Author for correspondence: Gilles Reysset. Tel: +33145688311. Fax: +33140613123.

Unité des Anaérobies, Institut Pasteur, 75724 Paris Cedex 15, France

\begin{abstract}
The genetic organization of two different 5-nitroimidazole (5-Ni) resistance genes was investigated: nimC and nimD from Bacteroides plasmids pIP419 and pIP421, respectively. The nimC gene (492 bp) and the nimD gene (495 bp) directed the synthesis of polypeptides with deduced molecular masses of $18.37 \mathrm{kDa}$ and $18.48 \mathrm{kDa}$, respectively. The predicted proteins showed $67-83 \%$ identity and $78-91 \%$ similarity with the products of two other nimA and nimB genes previously described and could be derived from a common ancestral gene. An insertion sequence element (IS1170) was identified upstream of the nimC gene. IS1170 is 1604 bp in length and is flanked by imperfect inverted repeats (15 bp). IS1170 is similar to the Bacteroides insertion sequence element IS942 with an identity of $70 \%$ at the nucleotide level. The single copy of IS1170 present on plasmid pIP419 is integrated 24 bp upstream of the initiation codon of nimC. Similar genetic organization was found on plasmid pIP421. One copy of another insertion sequence (1S1169) was found 4 bp upstream of the first ATG codon of the nimD gene. This element (1325 bp) shows a strong homology at the nucleotide level (70\% identity) with IS1186 and IS1168 found to be associated with the Bacteroides carbapenem resistance gene cfiA, and the 5Nirgenes nimA and nimB, respectively. There is strong evidence that, as in the case of the cfiA gene, the transcription of the four nim genes so far studied is directed by outward-oriented promoters, carried on the right ends of the different insertion sequence elements.
\end{abstract}

Keywords: Bacteroides, plasmids pIP419 and pIP421, 5-nitroimidazole resistance genes, insertion sequence elements

\section{INTRODUCTION}

Among the anaerobic bacteria involved in human infections, Bacteroides species are the most frequently isolated (Salyers, 1984). These bacteria are commonly resistant to antibiotics, particularly to many $\beta$-lactam antibiotics, tetracycline and macrolides, but remain sensitive to the 5nitroimidazoles. Despite the clinical use of these antimicrobial agents for more than 30 years, the incidence of

†Present address: Université Moulay Ismail, Faculté des Sciences, Département de Biologie, BP 4010, Méknes, Morocco.

Abbreviation: 5-Ni, 5-nitroimidazole.

The GenBank/EMBL/DDBJ accession numbers for the nucleotide sequences of the fragments from pIP419 and pIP421 are X76948 and X76949, respectively. 5-nitroimidazole (5- $\mathrm{Ni}$ ) resistance in Bacteroides is still very low.

The demonstration that specific transmissible genetic determinants conferring a low level of resistance occur in Bacteroides was reported in 1989 (Breuil et al., 1989). Two resistance determinants ( $\operatorname{nim} A$ and $\operatorname{nim} C$ ) were shown to be located on small mobilizable plasmids, designated pIP417 $(7.7 \mathrm{~kb})$ and pIP419 $(10 \mathrm{~kb})$ derived from Bacteroides vulgatus $\mathrm{BV} 17$ and Bacteroides thetaiotaomicron BT13, respectively (Reysset et al., 1992). Although differing in their restriction maps, the two plasmids showed homology in a region involved in their mobilization (Reysset et al., 1992). Another resistance determinant $(\operatorname{nim} B$ ) was found to be located on the chromosome of Bacteroides fragilis strain BF8 (Sebald et al., 1990; Haggoud et al., 1994). This determinant was also 
shown to be transferable homo- or heterospecifically through a conjugation-like process. Subsequently, another $5-\mathrm{Ni}^{\mathrm{r}}$ plasmid was found in three clinical isolates of Bacteroides (Reysset $e t$ al., 1993).

The $5-\mathrm{Ni}^{\mathrm{r}}$ genes of strain $\mathrm{BF} 8(\operatorname{nim} B)$ and of plasmid pIP417 (nim $A$ ) have been sequenced (Haggoud et al., 1994). The nucleotide sequences and the deduced amino acid sequences show about $70 \%$ identity suggesting that, although different, the two genes are closely related. Moreover, an identical insertion sequence element (IS1168, $1320 \mathrm{bp}$ ) was found a few base pairs upstream of the start codons of both genes (Haggoud et al., 1994). An identical insertion sequence element (IS1180) was shown to activate the carbapenemase gene $c f i A$ of $B$. fragilis when present immediately upstream of the gene (Podglajen $e t$ al., 1994). The transcription of cfi $A$ was found to be driven from a promoter identified on the right end of the insertion sequence element (Podglajen et al., 1994). The same promoter should be efficient in the transcription of the $\operatorname{nim} A$ and $\operatorname{nim} B$ genes.

There is therefore strong evidence that these newly described insertion sequences contain the regulatory signals for the expression of the resistance genes. However, our studies have indicated that IS1168 fails to hybridize with pIP419 and therefore transcription of nim genes is not always directed by IS1168. The aim of this work was to compare the four nim genes and their upstream regions.

\section{METHODS}

Bacterial strains and plasmids. The Escherichia coli strains used in this study were HB101 (Boyer \& Roulland-Dussoix, 1969), JM109 (Yanisch-Perron et al., 1985) and GT869 (Parsot, 1986). The B. fragilis strains were the plasmid-free strain BF-638R (Privitera et al., 1979) used as a recipient for transformation, and BF-F238, BF-F239 and BF-M6 (Reysset et al., 1993). The following plasmids were also used: pIP419 from B. thetaiotaomicron BT13 and the recombinant plasmid pFK100 (Reysset et al., 1992), pIP421 (7.3 kb) isolated from strain BF-F239, pUC19 (Yanisch-Perron et al., 1985) and pBI191 (Smith, 1985).

Media and culture conditions. Bacteroides strains were cultured in an anaerobic chamber (La Calhène, 78140 Velizy, France) in $5 \% \mathrm{H}_{2}, 5 \% \mathrm{CO}_{2}$ and $90 \% \mathrm{~N}_{2}$. TGY medium was used as basal medium (Privitera et al., 1979). Wilkins-Chalgren medium (Oxoid) was used for antibiotic susceptibility testing and plating of transformants. E. coli strains used as cloning hosts were cultivated aerobically in L-broth supplemented with ampicillin $\left(100 \mu \mathrm{g} \mathrm{ml}^{-1}\right)$ where appropriate.

Preparation and analysis of plasmid DNA. Small scale preparations of plasmid DNA were obtained from exponential phase cells $\left(\mathrm{OD}_{660} 0.6\right)$ by the alkaline lysis procedure (Birnboim \& Doly, 1979). The DNA was purified by isopycnic ethidium bromide- $\mathrm{CsCl}$ ultracentrifugation. DNA was analysed by horizontal electrophoresis on $0.7 \%$ agarose gels in TBE buffer

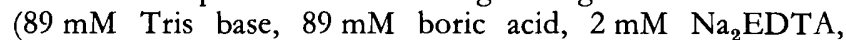
$\mathrm{pH} 8 \cdot 25)$. Restriction endonuclease analysis was performed under the conditions recommended by the suppliers (Amersham and Boehringer Mannheim).

Plasmid constructions and electrotransformation. All the recombinant plasmids used for cloning or phenotypic ex- pression in Bacteroides were constructed using the cloning vector pBI191 (Smith, 1985), whereas recombinant plasmids needed for DNA sequencing or in vitro transcription-translation assays were constructed using the cloning vector pUC19 (YanischPerron et al., 1985). Plasmids pFK110 and pFK111 are recombinant plasmids from pUC19 containing a $2 \mathrm{~kb}$ Sau $3 \mathrm{~A}$ restriction fragment from the $\operatorname{nim} C$ gene of pIP419 cloned in opposite orientations. Similarly, plasmids pFK310 and pFK311 contained the $1.8 \mathrm{~kb}$ HindIII restriction fragment from the nimD gene of pIP421 cloned in opposite orientations. In recombinant plasmids $\mathrm{pFK} 110$ and $\mathrm{pFK} 310$, the nim genes are located in the opposite orientation to the plac promoter. Electrotransformations of E. coli and Bacteroides were performed according to Dower et al. (1988) and Reysset et al. (1992) respectively.

DNA sequencing, sequence analysis, Southern blotting and DNA-DNA hybridization. DNA sequencing was performed by dideoxy sequencing reactions (Sanger et al., 1977) using modified T7 polymerase (Sequenase $2.0 \mathrm{kit}$, US Biochemical). Reaction mixtures were analysed by electrophoresis on 6 or $8 \%$ (w/v) polyacrylamide gels (Sambrook et al., 1989). Computer analysis was performed by using the Genetic Computer Group (GCG) programs (University of Wisconsin, Madison). The presence and copy number of IS1169 and IS1170 were determined by Southern blot analysis performed according to Bryant et al. (1988) on nylon $\mathrm{N}^{+}$membranes (Gene Screen ${ }^{+}$, Dupont de Nemours). Probes were purified restriction frag ments labelled with $\left[{ }^{32} \mathrm{P}\right] \mathrm{dATP}$ by nick translation (Boehringer Mannheim). The location of the $\mathrm{Ni}^{\mathrm{r}}$ determinant of plasmid pIP421 was checked using an internal probe of the nimB gene (IG probe) synthesized by PCR using the following oligonucleotide primers : 5'-ATGT'TTAGAGAAATGCG-3' and 5'GTCATTCCACAGTTCGT-3' (Haggoud et al., 1994). The amplified fragment was purified from agarose gel $(1 \%)$ with the Geneclean II kit (Gibco BRL) and radiolabelled as described previously.

In vitro transcription-translation. Recombinant plasmids pFK110, pFK111, pFK310 and pFK311, containing the pIP419 and pIP421 5- $\mathrm{Ni}^{\mathrm{r}}$ inserts cloned in opposite orientations, were subjected to transcription-translation in an in vitro prokaryotic DNA-directed system (Amersham). The proteins were separated by SDS-PAGE, and the ${ }^{35}$ S-labelled synthesized polypeptides detected by autoradiography.

\section{RES!JLTS}

\section{Location and nucleotide sequences of the 5-Nir determinants of plasmids pIP419 and pIP421}

The 5-Nir determinant encoded by plasmid pIP419 was previously cloned on vector pBI191 as a $2 \mathrm{~kb}$ Sau3A restriction fragment (Reysset et al., 1992) to give the plasmid pFK100. Deletion analysis of pFK100 was performed in order to locate precisely the $5-\mathrm{Ni}^{\mathrm{r}}$ gene, and the subclones were analysed for expression of $5-\mathrm{Ni}$ resistance in B. fragilis BF-638R. It was demonstrated that a recombinant plasmid containing the $1.25 \mathrm{~kb}$ EcoRI-Sau3A restriction fragment (Fig. 1) confers $5-\mathrm{Ni}$ resistance to susceptible Bacteroides strains, whereas the DraI-Sau3A and the Sau3A-PvuII restriction fragments (approx. 1 and $1.1 \mathrm{~kb}$, respectively) did not.

Plasmids $(7.3 \mathrm{~kb})$ detected in three $5-\mathrm{Ni}^{\mathrm{r}}$ clinical isolates of B. fragilis (strains BF-F238, BF-F239 and BF-M6), were 
(a)

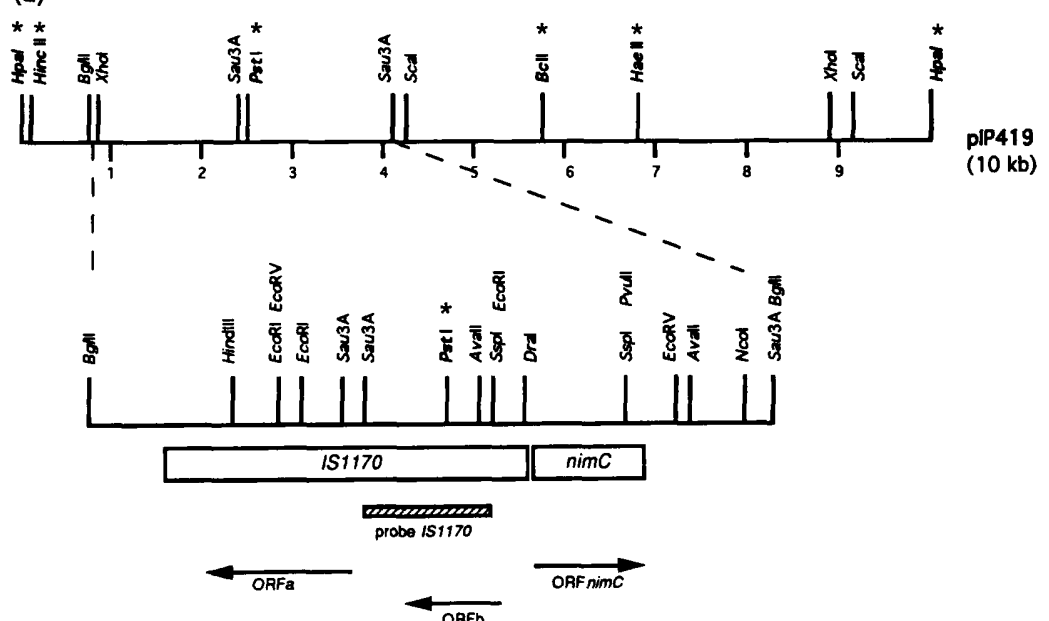

(b)

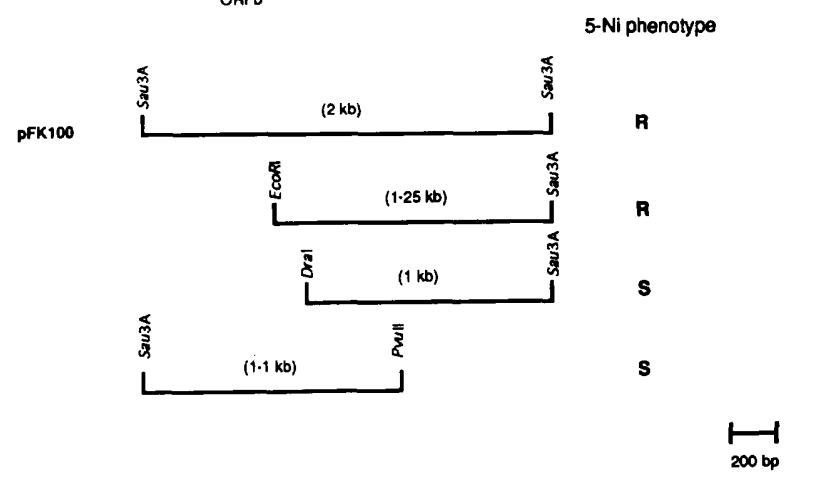

Fig. 1. Physical map and subclones of plasmid plP419 and the nimC region. (a) Physical map, with unique restriction sites indicated by asterisks. An expanded map of the sequenced region is also shown. The location and the putative direction of transcription of the ORFs ( $a$ and $b$ ) within the insertion sequence element IS1170, and the single ORF of the nimC gene are indicated by arrows. The location of IS1170 used as an internal probe in hybridization experiments is indicated by a hatched box. (b) Subclones of PFK100 and the corresponding 5 - $\mathrm{Ni}$ phenotypes in $\mathrm{B}$. fragilis $B F-638 R$. $R$, resistance; $S$, sensitivity.

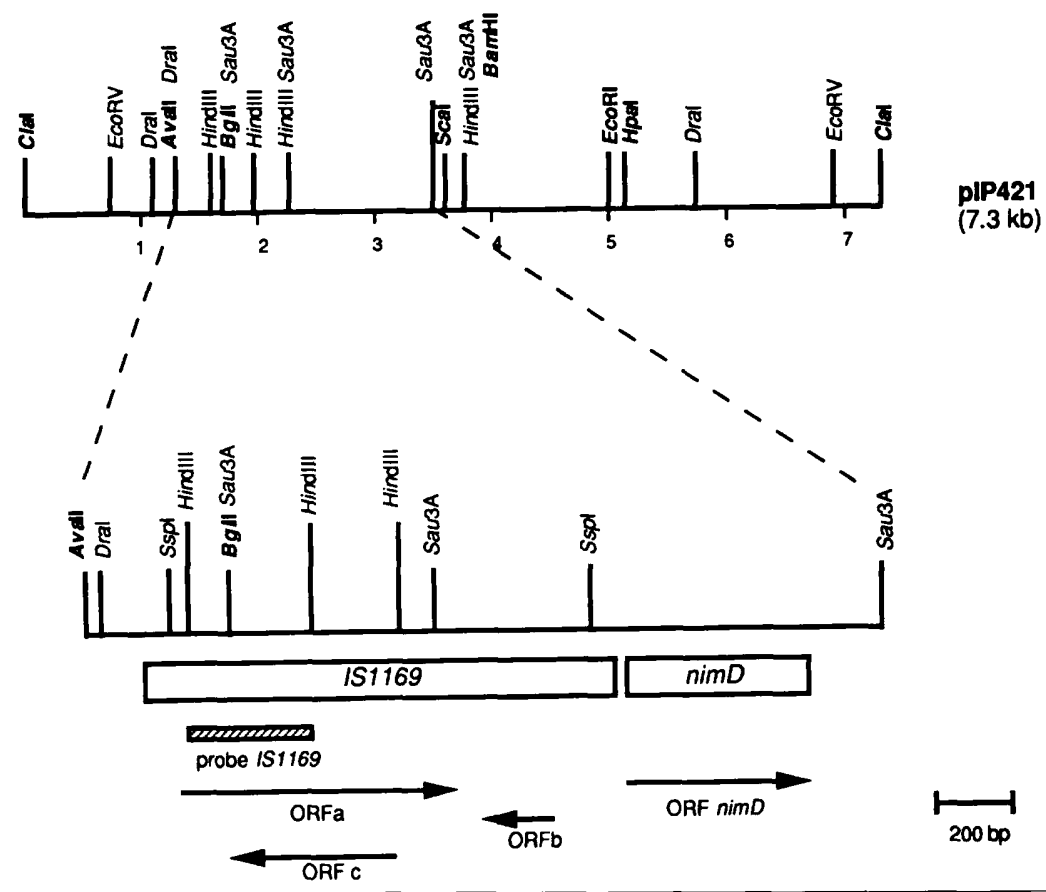

Fig. 2. Physical map of plasmid plP421 and the nimD region. The unique restriction sites are indicated in bold type. An expanded map of the sequenced region is also shown. The location and putative direction of transcription of the three major ORFs (a to c) within the insertion sequence element IS1169, and the single ORF of the nimD gene are indicated by arrows. The location of IS1169 used as an internal probe in hybridization experiments is indicated by a hatched box.

shown to confer resistance when transferred by conjugation or transformation to $B$. fragilis strain BF-638R (Reysset et al., 1993). Preliminary restriction analysis of the three plasmids with HindIII and PvuII enzymes has shown similar restriction patterns. However, these are distinct from those of the previously isolated $5-\mathrm{Ni}^{\mathrm{r}}$ 


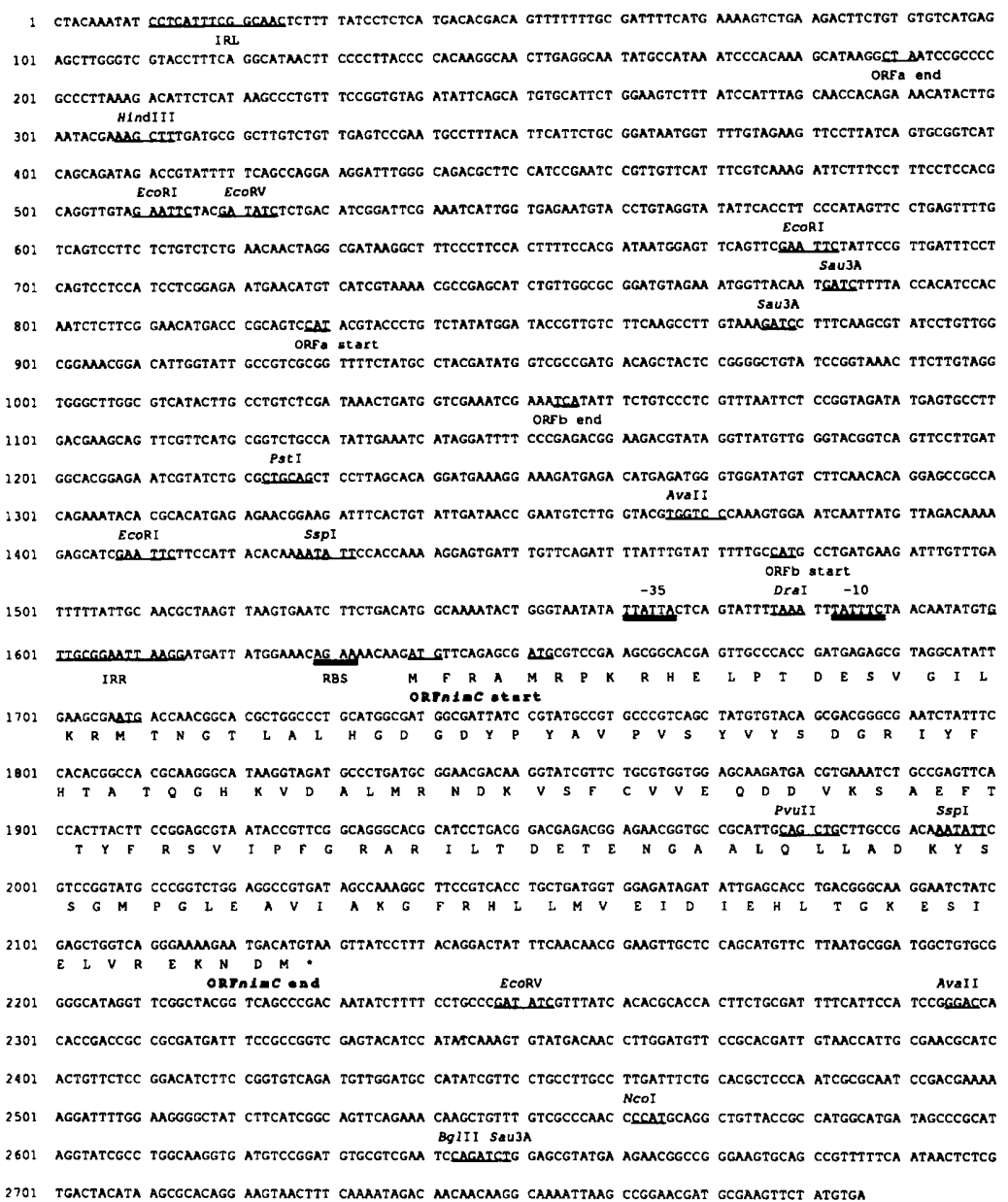

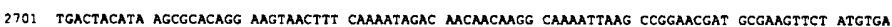

Fig. 3. Nucleotide sequence of the $\operatorname{nim} C$ region of plasmid pIP419. Relevant restriction sites are indicated below the sequence. The two inverted repeat sequences of IS1170 [IRL (left) and IRR (right)] are indicated by lines below the corresponding nucleotides. The position of the start and stop codons of ORFa and ORFb within the IS element is also underlined. The three possible ATG start codons at nt positions 1639, 1651 and 1708 (underlined) and the stop codon TAA at nt 2130 (indicated by an asterisk) of ORFnimC (labelled in bold type) are shown. The predicted amino acid sequence for nimC is shown under the sequence, starting at nt 1639. The putative ribosome-binding site (RBS) and promoter sequences of ORFnimC are double-underlined.

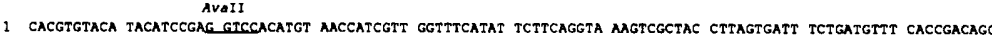

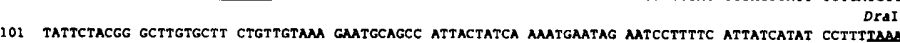

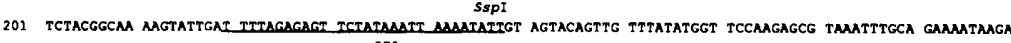
IRL

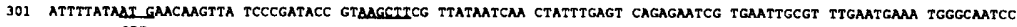
ORFo start BgIII moI

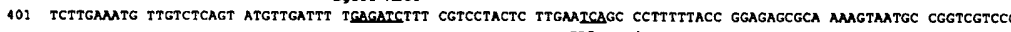

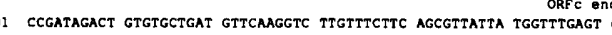

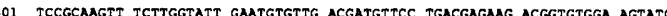
HindIII

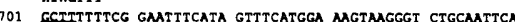

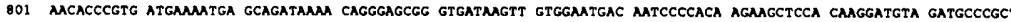

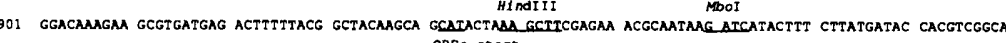

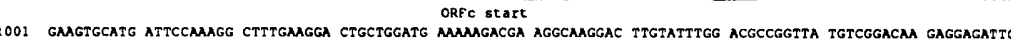

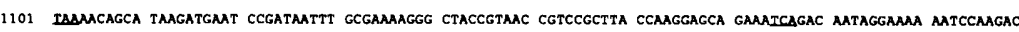
ORFa end

1201 ACGTTGCCTT GTCGRCCATG TATTCGGGT TGAGGAMGM ACGATCCTTE GACTTGTGCT GCTACACTA GCGCTTATTC GTGCTAMAGC CAMTGTAGCA

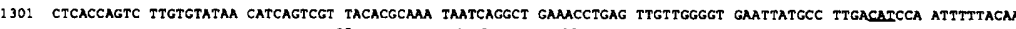

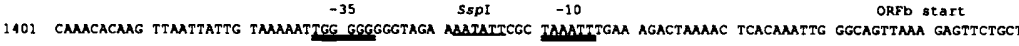

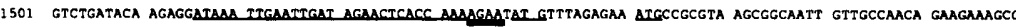

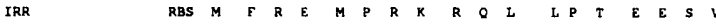
ORTnSAD atex

1601 TTGCCATTCT TGAACGGATG ACAAACGGGA CGTTGGCTCT TCATGGGGAT GACGGCTATC CGTATGCCGT CCCTGTCAGT tatGTATATG CCGATGGCAA

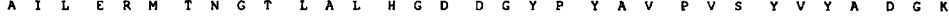

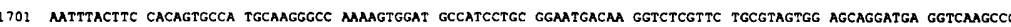
I Y F H S A M O G P K V D A I L R N D K V S F C V V E O D E V K P

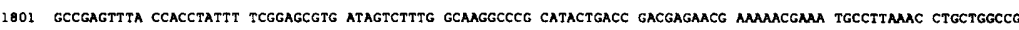
A E

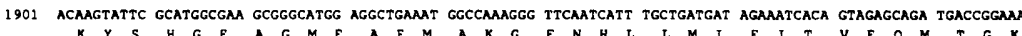
K Y Y $S$ S

2001 AGAAGCCATC GAACTGACAA GGGGAAGAM CGGATGTTCT TCACAAAGAA TGGCTGACTG ACGGAGAAAA MAAGCTGTTT CGCGTCCGCG

E A I
}

Fig. 4. Nucleotide sequence of the nimD region of plasmid pIP421. Relevant restriction sites (see Fig. 2) are indicated below the sequence. The two inverted repeat sequences of IS1169 [IRL (left) and IRR (right)] are indicated by lines below the corresponding nucleotides. The position of the start and stop codons of ORFa, b and $c$ within the IS element is also underlined. The three possible ATG start codons at nt positions 1549, 1561 and 1618 (underlined) and the stop codon TGA at nt 2043 (indicated by an asterisk) of ORFnimD (labelled in bold type) are shown. The predicted amino acid sequence for nimD is shown under the sequence. The putative ribosome-binding site (RBS) and promoter sequences of ORFnimD are doubleunderlined. 
plasmids. This result was confirmed by examining the physical map of plasmid pIP421 of strain BF-F239. Restriction enzyme sites were identified and localized by analysis of DNA cleaved by various enzymes (unique and double digestions). The linear map of pIP421 is given in Fig. 2. Plasmid pIP421 had unique restriction sites for AvaII, BamHI, BglII, ClaI, EcoRI, HpaI and ScaI, two sites for EcoRV, and three sites for DraI. Plasmid pIP421 was not digested by the following restriction enzymes: AvaI, HaeII, KpnI, NcoI, NsiI, PvuII, SalI, SmaI and XbaI. The location of the 5-Nir determinant of plasmid pIP421 was then determined by Southern blot analysis, using the probe IG defined in Methods. Strong hybridization was only observed with a $1.8 \mathrm{~kb}$ HindIII restriction fragment (data not shown).

Both $5-\mathrm{Ni}^{\mathrm{r}}$ determinants, and the adjacent regions, were then sequenced from both strands after subcloning into pUC19. The complete nucleotide sequences of the pIP419 and PIP421 5-Ni resistance regions are given in Figs 3 and 4 , respectively. The location of the ORFs (ORFnimC and ORFnimD) corresponding to the $5-\mathrm{Ni}^{\mathrm{r}}$ determinants of plasmids pIP419 and pIP421, respectively, was determined and compared with the nucleotide sequences of the $\operatorname{nim} A$ and $\operatorname{nim} B$ genes (Haggoud et al., 1994). As already observed in the case of the $5-\mathrm{Ni}^{\mathrm{r}}$ determinants cloned from plasmid pIP417 and from the chromosome of strain BF8, the two ORFs (492 and $495 \mathrm{bp}$, respectively) have three possible ATG start codons (Figs 3 and 4).

\section{The nim gene products}

The largest ORF's, ORFnimC and ORFnimD, may encode polypeptides of 163 and 164 amino acid residues (molecular mass 18.37 and $18.48 \mathrm{kDa}$ ), respectively. Pairwise comparisons of the deduced amino acid sequences of the polypeptides encoded by the four nim genes so far sequenced were done with the Gap program of the GCG package. The polypeptides show $67-83 \%$ identity, and $78-91 \%$ similarity. The higher values were obtained with the polypeptides encoded by the chromosomal $\operatorname{nimB}$ gene of strain BF8 and those of the nimD gene of plasmid pIP421. No significant homology was found between the nucleotide and predicted amino acid sequences for the nim genes and those available in the GenBank, EMBL or SWISS-PROT databases.

An E. coli in vitro transcription--translation system was used to investigate the polypeptide synthesis directed by the $2 \mathrm{~kb}$ Sau $3 \mathrm{~A}$ and the $1.8 \mathrm{~kb}$ HindIII $5-\mathrm{Ni}^{\mathrm{r}}$ inserts of plasmids pIP419 and pIP421, respectively. The two restriction fragments were cloned in both orientations into pUC19. The results are shown in Fig. 5. In all cases, polypeptides of the expected molecular mass were synthesized from the genes cloned in both orientations, suggesting that the promoters of both genes were functional in E. coli. The molecular masses of NimC and NimD determined on polyacrylamide gels were 19 and $17 \mathrm{kDa}$, respectively, in agreement with the predicted

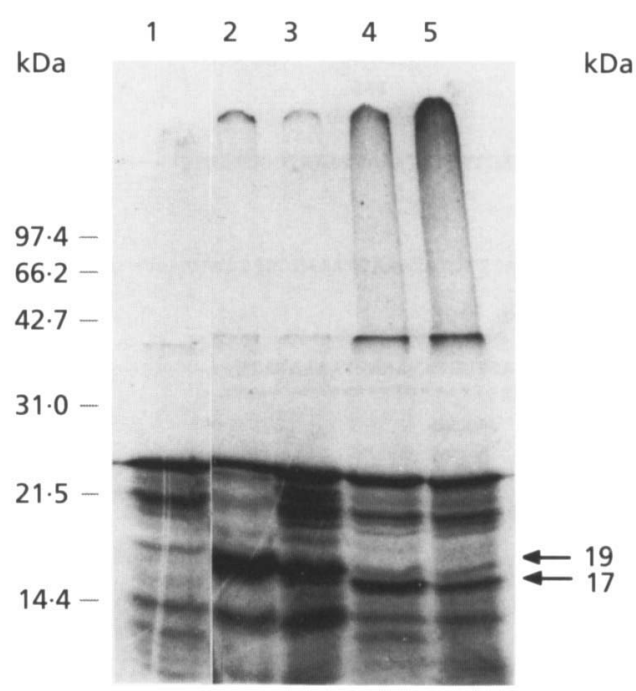

Fig. 5. Autoradiograph of plasmid-encoded proteins detected in an in vitro $E$. coli transcription-translation assay. The $\left.{ }^{35} \mathrm{~S}\right]$ methionine-labelled proteins were separated on an SDS polyacrylamide gel (15\%, w/v, acrylamide). Lanes: 1, pUC19; 2 , pFK110; 3, pFK111; 4, pFK310; 5, pFK311. Standard proteins are indicated on the left. Products of the $\operatorname{nim} C$ and $\operatorname{nim} D$ genes are indicated by arrows.

theoretical values of 18.37 and $18.48 \mathrm{kDa}$. Similar results were obtained using the $\operatorname{nim} A$ and $\operatorname{nim} B$ cloned genes (data not shown).

\section{Characterization of an insertion sequence element, IS1170, present upstream of the nimC gene}

Analysis of the nucleotide sequence upstream of the nim $C$ gene of plasmid pIP419 revealed the presence of an insertion sequence element (IS1170). IS1170 (1604 bp) extends from nucleotide 10 to 1614 (Fig. 3) and is located 24 bp upstream of the first ATG codon of $\mathrm{nimC}$. IS1170 is flanked by $15 \mathrm{bp}$ imperfect inverted repeats (IRL and IRR) $12 \mathrm{bp}$ of them matching. The $\mathrm{G}+\mathrm{C}$ content of $\operatorname{IS} 1170(42.8 \mathrm{~mol} \%)$ is within the range (39-46 $\mathrm{mol} \%)$ determined for genomic DNAs of Bacteroides spp. (Johnson, 1978). Two ORFs were present on the same strand within IS1170. They extend from nucleotide 830 to 189 and from nucleotide 1479 to 1054 and could code for polypeptides of 141 and 213 amino acids, respectively. A search in the databases for amino acid sequences related to the two ORFs revealed an identity of 70 and $76 \%$, respectively, with both ends of the unique ORF of Bacteroides IS 942 described by Rasmussen \& Kovacs (1991), suggesting that mutations occurred in the ancestral sequence of the gene encoding the putative transposase. Comparison of the nucleotide sequences of IS 942 and IS 1170 revealed that they show $70 \%$ identity, the two inverted repeats of IS 1170 and IS 942 being more conserved. The left and right IR of IS 942 and IS 1170 show 80 
(a)

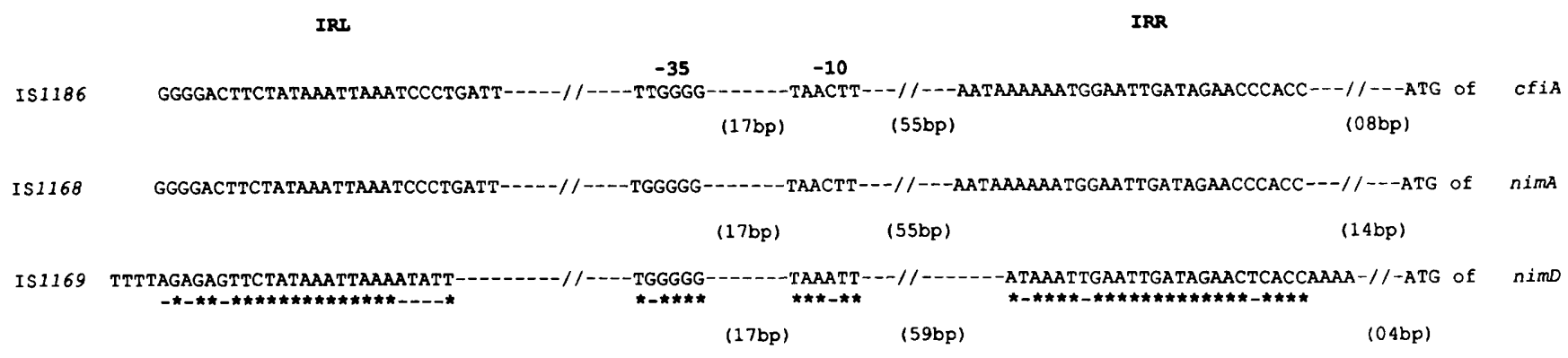

(b)

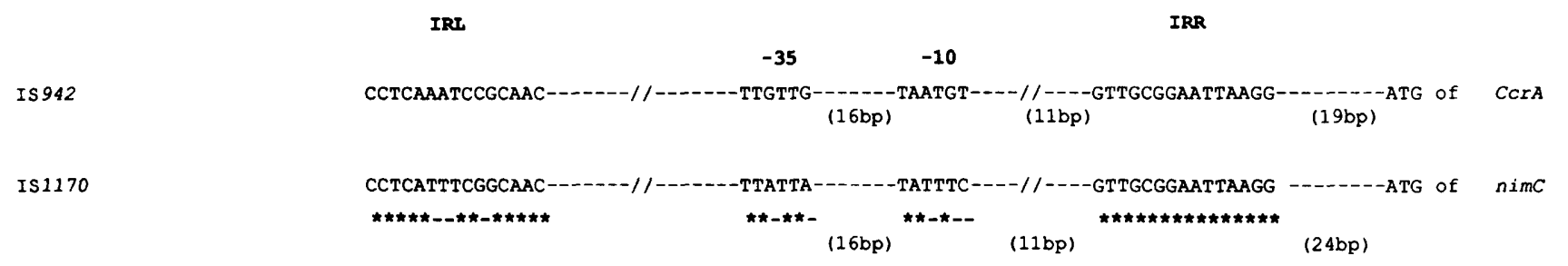

Fig. 6. Comparison of the nucleotide sequences of the inverted repeat left (IRL) and right (IRR), and the outwardoriented promoter of the two insertion sequence families. (a) Comparison of IS1186 (Podglajen et al., 1994; GenBank accession number X72301); IS1168 (Haggoud et al., 1994; GenBank accession number X71444) and IS1169 (this work). (b) Comparison of IS942 (Rasmussen et al., 1991; GenBank accession number J03326) and IS1170 (this work). The figures in parentheses indicate the distances between the regions. Nucleotide identity is indicated by asterisks and mismatches by dashes.

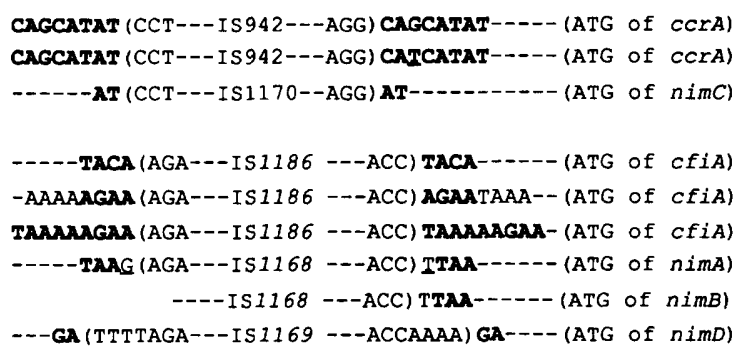

Fig. 7. Comparison of the insertion site duplication sequences of different Bacteroides IS elements upstream of the ccrA, cfiA and nim genes. The insertion site duplication sequences are written in bold type. Data are from Rasmussen \& Kovacs (1991) for IS942, Podglajen et al. (1994) for IS1168 and Haggoud et al. (1994) for IS1168. Other data are from this work.

and $100 \%$ identity respectively (Fig. 6). A putative duplication of the target site (Galas \& Chandler, 1989), created by the insertion of IS1170 upstream of the nimC gene, was only $2 \mathrm{bp}$ in length (AT) instead of the $8 \mathrm{bp}$ target duplication found by Rasmussen \& Kovacs (1991) for two integration events of IS 942 upstream of the $\operatorname{crr} A$ gene (Fig. 7).

\section{Characterization of an insertion sequence element, IS1169, present upstream of the nimD gene}

Sequence analysis revealed the presence of another insertion sequence element (IS1169) 4 bp upstream of the first ATG codon of the nimD gene. IS1169 (1325 bp)
$(\mathrm{G}+\mathrm{C} 41 \mathrm{~mol} \%)$ contains imperfect inverted repeat ends of $29 \mathrm{bp}$ and shows strong homology with IS1168 adjacent to the $\operatorname{nim} A$ and $\operatorname{nim} B$ genes, both in the IR and ORF regions. The IRL and IRR of IS1169 show 62 and $76 \%$ identity, respectively, to the corresponding regions of IS1168 (Fig. 6). The three ORFs (ORFa, b and c) found within IS 1169 potentially code for basic polypeptides of molecular mass $31.57,8.17$ and $18.34 \mathrm{kDa}$, respectively. The largest ORF (ORFa) shows $78.5 \%$ identity at the amino acid level with the corresponding ORF of IS1168. The results of a search in the GenBank and EMBL databases for sequences related to IS1169 were identical to those found for IS1168 (Haggoud et al., 1994), i.e. IS1246 harboured by plasmid pWWO of Pseudomonas putida (Reddy et al., 1994, accession number L11583), IS1106 from Neisseria meningitidis (Knight et al., 1992), IS 5 from IKH100 (Kröger \& Hobom, 1982) and IS52 from Pseudomonas syringae (Yamada et al., 1986).

The duplication of the target site expected for the insertion sequence of the IS 5 family is $4 \mathrm{bp}$ in length (Galas \& Chandler, 1989). Some examples of the target duplication of IS1186, IS1168 and IS1169 are illustrated in Fig. 7. For IS1186, Podglajen et al. (1994) found in all cases but one a $4 \mathrm{bp}$ target duplication. In one strain the target duplication was $9 \mathrm{bp}$ in length. The situation for IS1168 present upstream of the nim genes is less clear. In the copy of IS1168 present upstream of the nim A gene a 3 bp target duplication (TAA) was found, separated by one nucleotide from both ends of the inverted repeats. A similar situation was observed by Knight et al. (1992) for IS1106 
(a)

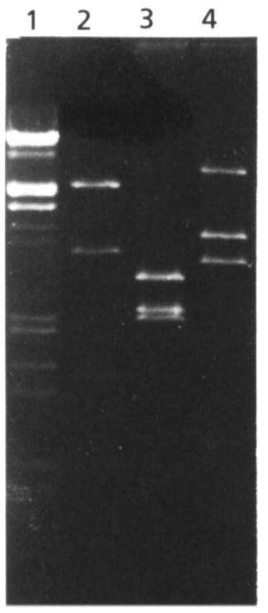

(b)

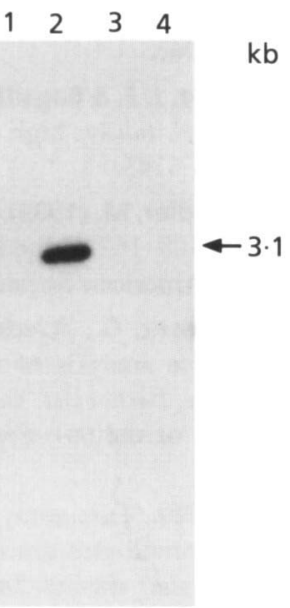

(c)

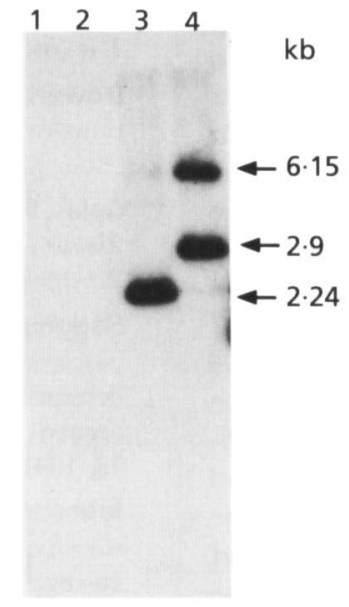

Fig. 8. Southern blot analysis of digested DNA of pIP419 and pIP421 using IS1170 and IS1169 as internal probes. (a) Plasmid DNA restriction profiles on stained agarose gel; (b) Southern analysis of the gel using the IS1170 probe; (c) Southern analysis of the gel using the IS1169 probe. Lanes: 1, $\lambda$ DNA digested by HindIII and EcoRV (molecular mass standard); 2, pIP419 digested by Dral; 3. plP421 digested by Dral-Scal; 4, plP421 digested by ECORV-Scal (note that a $6.15 \mathrm{~kb}$ restriction fragment which also hybridizes with the 151169 probe is from a partial digestion fragment of the plasmid consisting of the 3.25 plus $2.9 \mathrm{~kb}$ restriction fragments). in N. meningitidis. For the copy of IS1169 present upstream of the nimD gene, a 2 bp sequence (GA) was found as a potential target duplication site.

\section{Absence of a composite transposon-like structure on plasmids pIP419 and pIP421}

It has been previously shown that only one copy of IS 1168 is present on plasmid pIP417, demonstrating that the nim $A$ gene is not part of a transposon (Haggoud et al., 1994). The copy number of IS 1170 and IS1169 from plasmids pIP419 and pIP421, respectively, was determined by Southern blot analysis. The probes used in this study were the internal Sau3A-EcoRI restriction fragment of length $626 \mathrm{bp}$ (nt 782-1408, Fig. 3) for IS1170, and the internal HindIII restriction fragment of length $366 \mathrm{bp}$ (nt 333-699, Fig. 4) for IS1169. The location of the two probes are shown in Figs 1 and 2, respectively. Plasmid pIP419 was digested by DraI to give restriction fragments of lengths $3 \cdot 1,5.5$ and $1.5 \mathrm{~kb}$. Plasmid pIP421 was doubly digested by $S_{c a} \mathrm{I}-D r a \mathrm{I}$ and $S_{c a} \mathrm{I}-E_{c o} \mathrm{RV}$ to give restriction fragments of lengths $2 \cdot 24,2 \cdot 1,2 \cdot 65$ and $0 \cdot 26 \mathrm{~kb}$, and $2 \cdot 9$, 3.25 and $1.1 \mathrm{~kb}$, respectively. Results of hybridization experiments are shown in Fig. 8. For plasmid pIP419, only the expected $2.95 \mathrm{~kb}$ DraI restriction fragment hybridized with the IS1170 probe (lane 2) but not with the IS 1169 probe. For plasmid pIP421, the $2 \cdot 24 \mathrm{~kb}$ ScaI-DraI (lane 3) and the $2.9 \mathrm{~kb}$ EcoRV-ScaI (lane 4) restriction fragments hybridized with the IS1169 probe. From the physical maps we concluded that in both cases only one copy of the insertion sequence element is present upstream of each nim gene. Thus, the insertion sequence elements of plasmids PIP419 and PIP421 are not parts of a composite transposon.

\section{DISCUSSION}

The two 5-Nir genes nim $C$ and nimD, present on plasmids pIP419 and PIP421, have been cloned and sequenced. Nucleotide sequence analysis revealed the presence of two ORFs, ORFnimC and ORFnimD, which may encode polypeptides of 163 and 164 amino acid residues (mo- lecular mass 18.37 and $18.48 \mathrm{kDa}$ ), respectively. The two predicted proteins show $72.5 \%$ identity and $84.7 \%$ similarity.

The four nim genes so far studied (this work, and Haggoud et al., 1994) share some common features. The nucleotide sequence length is from 492 to $534 \mathrm{bp}$ with a $\mathrm{G}+\mathrm{C}$ content varying from 42.8 to $55.9 \mathrm{~mol} \%$. Pairwise comparisons of the nucleotide sequences reveal an identity of about $70 \%$, which suggests that the nim genes are closely related. Nevertheless, the multiple alignment of the four sequences reveals that they show approximately $49 \%$ identity, indicating a high degree of variability at the nucleotide level. The genes could direct the synthesis of polypeptides of molecular mass $18-20 \mathrm{kDa}$ showing $67-83 \%$ identity and $78-91 \%$ similarity when the conservative amino acid substitutions are taken into account. The multiple alignment of the four amino acid sequences disclosed a greater homology of the nim gene products, with an identity of $57 \%$ and a similarity of $78 \%$. We suggest that the four genes, whether chromosomally determined or plasmid-borne, are derived from a common ancestral gene. No significant homology was found with either the nucleotide or amino acid sequences in the GenBank and EMBL databases.

Another important feature is that each nim gene is preceded by an insertion sequence element located a few base pairs upstream of the putative start codon. The same insertion sequence element, IS1168, was found upstream of the $\operatorname{nim} A$ and $\operatorname{nim} B$ genes, whereas other insertion sequences, IS1169 and IS1170, were found upstream of the nimD and nim $C$ genes, respectively. These insertion sequence elements belong to two families as indicated in Fig. 6.

The first family consists of the three Bacteroides insertion sequence elements IS1168, IS1169 and IS1186. The latter element was detected upstream of the carbapenemase gene cf $A$ in a clinical isolate of $B$. fragilis (Podglajen et al., 1994). In this carbapenem-resistant strain, the transcription of cfi $A$ was found to be driven from a promoter identified on the right end of IS1186. The -35 region 
sequence ('T'TGGGG) is separated by the optimal spacing distance of $17 \mathrm{bp}$ from the -10 region (TAACTT). Comparison of the nucleotide sequence of IS1168 and IS1186 revealed that the two insertion sequence elements were almost identical ( $99 \cdot 7 \%$ identity), suggesting that the transcription of the $\operatorname{nim} A$ gene is also directed by the same promoter. Furthermore, analysis of the nucleotide sequence of IS1169, which shows $71 \%$ identity to IS1168, reveals that there is a nearly perfect match (five out of six base pairs) between both the -35 and -10 sequences of IS1186 (see Fig. 6). We thus conclude that, as for the cfi $A$ gene, the $\operatorname{nim} A, B$ and $D$ genes are transcribed from an internal insertion sequence promoter. In a survey of 365 $\mathrm{Ni}^{\mathrm{r}}$ clinical isolates of Bacteroides strains, the insertion sequence elements of the first family were found to be associated with a nim gene in $94 \%$ of the strains (Reysset et al., 1993, and data not shown), which suggests a wide distribution amongst resistant strains.

IS942 and IS 1170 belong to a second Bacteroides insertion sequence family. One copy of IS 942 was found integrated $19 \mathrm{bp}$ upstream of the predicted initiation codon of the metallo- $\beta$-lactamase gene $\operatorname{cor} A$ cloned from $B$. fragilis TAL3636 (Rasmussen \& Kovacs, 1991). The authors have also suggested that the transcription of the $\operatorname{cr} A$ gene was driven from a promoter within the IS element, and have defined the -35 and -10 promoter-like sequences (Rasmussen et al., 1990). As shown in Fig. 7, two related -35 and -10 boxes were also found in the corresponding regions of IS1170. Moreover, although the $1 \mathrm{~kb}$ DraISau3A restriction fragment of plasmid pIP419 contained the entire ORFnimC, it did not confer the $5-\mathrm{Ni}^{\mathrm{r}}$ phenotype, the DraI site being located between the proposed -35 and -10 sequences (see Fig. 3 ).

An identical tetranucleotide AGAA sequence, which appeared to be an efficient ribosome-binding site in Bacteroides (Podglajen et al., 1994), was found between the end of the IRR of each insertion sequence element and the ATG start codon of the $c$ fi $A, \operatorname{cr} A$ or nim genes. We have also shown by in vitro transcription-translation assay that the promoters of IS1168, IS1169 and IS1170 are functional in E. coli, suggesting that the outward-oriented promoters of the two Bacteroides IS families could be functional in this species.

\section{ACKNOWLEDGEMENTS}

We thank S. Azoulay-Nizard for excellent technical assistance and $\mathrm{F}$. Jackson for critical reading of the manuscript.

\section{REFERENCES}

Birnboim, H. C. \& Doly, J. (1979). A rapid alkaline extraction procedure for screening recombinant plasmid DNA. Nucleic Acids Res 7, 1513-1522.

Boyer, H. W. \& Roulland-Dussoix, D. (1969). A complementation analysis of the restriction and modification of DNA in E. coli.J Mol Biol 41, 459-472.

Breuil, J., Dublanchet, A., Truffaut, N. \& Sebald, M. (1989). Transferable 5-nitroimidazole resistance in the Bacteroides fragilis group. Plasmid 21, 151-154.
Bryant, D. A. \& Tandeau De Marsac, N. (1988). Isolation of genes encoding components of photosynthetic apparatus. Methods Enzymol 167, 755-765.

Dower, W. J., Miller, J. F. \& Ragsdale, C. W. (1988). High efficiency transformation of E. coli by high voltage electroporation. Nucleic Acids Res 16, 6127-6145.

Galas, D. J. \& Chandler, M. (1989). Bacterial insertion sequences. In Mobile DN A, pp. 109-162. Edited by D. E. Berg \& M. M. Howe. Washington DC: American Society for Microbiology.

Haggoud, A., Reysset, G., Azeddoug, H. \& Sebald, M. (1994). Nucleotide sequence analysis of two 5-nitroimidazole resistance determinants from Bacteroides strains and of a new insertion sequence upstream of the two genes. Antimicrob Agent Chemother 38, 1047-1051.

Johnson, J. L. (1978). Taxonomy of the Bacteroides. I. Deoxyribonucleic acid homologies among Bacteroides fragilis and other saccharolytic Bacteroides species. Int J Syst Bacteriol 28, 245-256.

Knight, A. I., Ni, H., Cartwright, K. A. V. \& McFadden, J. J. (1992). Identification and characterization of a novel insertion sequence, IS1106, downstream of the por $A$ gene in B15 Neisseria meningitidis. Mol Microbiol 6, 1565-1573.

Kröger, M., \& Hobom, G. (1982). Structural analysis of insertion sequence IS5. Nature 297, 159-162.

Parsot, C. (1986). Evolution of biosynthetic pathways: a common ancestor for threonine synthase, threonine dehydratase and $\mathrm{D}$-serine dehydratase. EMBO J 5, 3013-3019.

Podglajen, I., Breuil, J. \& Collatz, E. (1994). Insertion of a novel DNA sequence, IS1186, upstream of the silent carbapenemase gene cf $A$, promotes expression of carbapenem resistance in clinical isolates of Bacteroides fragilis. Mol Microbiol 12, 105-114.

Privitera, G., Dublanchet, A. \& Sebald, M. (1979). Transfer of multiple antibiotic resistance between subspecies of Bacteroides fragilis. J Infect Dis 139, 97-101.

Rasmussen, B. A. \& Kovacs, E. (1991). Identification and DNA sequence of a new Bacteroides fragilis insertion sequence-like element. Plasmid 25, 141-144.

Rasmussen, B. A., Gluzman, Y. \& Tally, F. P. (1990). Cloning and sequencing of the class $\mathrm{B} \beta$-lactamase gene $(\mathrm{cr} A)$ from Bacteroides fragilis TAL3636. Antimicrob Agents Chemother 34, 1590-1592.

Reddy, B. R., Shaw, E., Sayers, J. R. \& Williams, P. A. (1994). Two identical copies of IS1246, a 1275 base pair sequence related to other bacterial insertion sequences, enclose the $x y$ lgenes of TOL plasmid pWWO. Microbiol 141, 2305-2307.

Reysset, G., Haggoud, A., Su, W. J. \& Sebald, M. (1992). Genetic and molecular analysis of pIP417 and pIP419: Bacteroides plasmids encoding 5-nitroimidazole resistance. Plasmid 27, 181-190.

Reysset, G., Haggoud, A. \& Sebald, M. (1993). Genetics of resistance of Bacteroides species to 5-nitroimidazole. Clin Infect Dis 16 (Suppl), S401-S403.

Salyers, A. A. (1984). Bacteroides of the human lower intestinal tract. Annu Rev Microbiol 38, 293-313.

Sambrook, J., Fritsch, E. F. \& Maniatis, T. (1989). Molecular Cloning: a Laboratory Manual, 2nd edn. Cold Spring Harbor, NY: Cold Spring Harbor Laboratory.

Sanger, F., Nicklen, S. \& Coulson, A. R. (1977). DNA sequencing with chain-terminating inhibitors. Proc Natl Acad Sci USA 74, 5463-5467.

Sebald, M., Reysset, G. \& Breuil, J. (1990). What's new in 5-nitroimidazole resistance in the Bacteroides fragilis group? In Clinical and Molecular Aspects of Anaerobes, pp. 217-225. Edited by S. P. Borriello. Petersfield: Wrightson Biomedical. 
Smith, C. J. (1985). Development and use of cloning systems for Bacteroides fragilis: cloning of a plasmid-encoded clindamycin resistance determinant. J Bacteriol 164, 294-301.

Yamada, T., Lee, P.-D. \& Kosuge, T. (1986). Insertion sequence elements of Pseudomonas savastanoi: nucleotide sequence and homology with Agrobacterium tumefaciens transfer DNA. Proc Natl Acad Sci US A 83, 8263-8267.
Yanisch-Perron, C., Vieira, J. \& Messing, J. (1985). Improved M13 phage cloning vectors and host strains : nucleotide sequences of the M13mp18 and pUC19 vectors. Gene 33, 103-119.

Received 24 August 1994; revised 21 November 1994; accepted 29 November 1994. 